\title{
Exploring dependence between brain signals in a monkey during learning
}

\author{
Cristina Gorrostieta a , Hernando Ombao ${ }^{a, b}, *,+$, Raquel Pradoc, Shaun Patel ${ }^{\mathrm{d}}$ \\ and Emad Eskandar ${ }^{\mathrm{d}}$
}

Our goal is to investigate dependence between brain wave oscillations in the nucleus accumbens (NAc) and the hippocampus $(\mathrm{Hc})$ regions of a macaque monkey during a learning association task. The classical approach to studying dependence in the spectral domain is via cross-coherence. It is computed for each frequency (or band) and identifies the frequency bands that drive the linear association between the components in a multi-variate time series. However, cross-coherence may not fully capture the complex dependence structure in brain signals such as local field potentials. In this article, we develop new tools for discovering associations between the theta (4-8 $\mathrm{Hz}$ ) and gamma (32-50 Hz) activities at both contemporaneous blocks and lagged time blocks. We propose a class of piecewise harmonizable processes under which we give a precise definition of these dependence measures and develop simple estimators in the case where the time-series data are recorded over several replicated trials. Our analysis clearly demonstrates strong dependence between the theta and gamma oscillations in the NAc and the Hc regions of a macaque monkey during learning. Moreover, we determined the lagged dependence that differentiate the 'correct' responses (i.e., the monkey was able to identify the correct association) from the 'incorrect' responses.

Keywords: Bivariate time series; cross-coherence; dual frequency coherence; coherence between amplitudes; Fourier transform; harmonizable processes; lagged coherence; Loève spectrum; spectral analysis.

\section{INTRODUCTION}

Spectral analysis is a widely used tool for studying associations between neuronal activity and cognition. Among the more recent work on this subject, see Bramon et al. (2004), Fiecas and Ombao (2011) Fiecas et al. (2010,), Sun et al. (2004) and Trejo et al. (2007). Within the neuroscience community, there is a strong interest in studying dependence between oscillatory activities at different brain regions. One standard approach to measuring dependence between channels or brain structures is by coherence analysis, which was demonstrated by Ombao and Van Bellegem (2008) to have an intuitive interpretation as being the squared magnitude of cross-correlation between a pair of bandpass-filtered time series. Estimation and inference for coherence is discussed by Brillinger (1981, 2001) and Shumway and Stoffer (2011).

The primary aim in this article is to investigate connectivity between oscillatory activities in the nucleus accumbens (NAc) and the hippocampus (Hc) of a macaque monkey during a learning experiment. These structures are implicated in human neurological and mental disorders. Here, we analyse local field potentials (LFPs) from chronically implanted probes. Unlike signals recorded from noninvasive procedures (such as electroencephalograms), LFPs directly measure brain electrical activity. During this experiment, the monkey learns the correspondence between elements in two groups of pictures. When the monkey correctly identified the correspondence in a trial, he received a reward in the form of a beverage. This research is clinically significant because temporal relationships between oscillatory patterns in the Hc and NAc during learning could potentially shed important insights on illnesses such as Alzheimer disease. Consequently, this could aid clinicians in generating rational strategies for modifying this process through deep brain stimulation or other means to enhance memory when it is affected by disease.

The common approach to study relationships in oscillatory patterns, standard coherence analysis, has a number of limitations. First, it examines dependence between brain waves oscillating only at identical frequency bands. This shortcoming is significant because recent studies suggest that memory and learning processes are directly related with interactions between waves oscillating at different frequency bands. See Nyhus and Curran (2010), Händel and Haarmeier (2009) and Varela et al. (2001). These results highlight the importance of developing statistical approaches for the analysis of such oscillatory dependencies, known as cross-frequency coupling. Another limitation of standard coherence is that it reflects only contemporaneous dependence (i.e. dependence at the same time block). Here, we develop a model that can directly address whether the presence of theta (4-8 Hz) activity in the NAc channel at the current time block leads to increased or decreased gamma (30-50 Hz) activity at the Hc channel at a next time block.

\footnotetext{
${ }^{a}$ Brown University

${ }^{b}$ University of California at Irvine

${ }^{c}$ University of California at Santa Cruz

${ }^{d}$ Massachusetts General Hospital

${ }^{*}$ Correspondence to: Hernando Ombao, Department of Statistics, Donald Bren School of Information and Computer Sciences, University of California, Irvine, Bren Hall 2019 Irvine, CA 92697-1250, USA

${ }^{\dagger}$ E-mail: hombao@uci.edu
} 
The remainder of this article is organized as follows. In Section 2, we discuss the concept of dual-frequency coherence (dependence between oscillatory activities at different frequency bands) under the context of harmonizable processes. In Section 3 , we develop the piecewise harmonizable process model which allows one to model both the evolution of the dual-frequency dependence and the dual-frequency dependence between different time blocks. In Section 4, we derive the estimators for the proposed spectral exploratory data analysis. In Section 5, we study cross-frequency oscillatory dependence in the LFP dataset from a macaque monkey using the proposed piecewise harmonizable process.

\section{OVERVIEW OF HARMONIZABLE PROCESSES}

The classical approach to studying spectral dependencies is via cross-coherence analysis. Define $\mathbf{U}(t)=[X(t), Y(t)]^{\prime}$ to be zero-mean bivariate stationary time series whose Cramér representation is given by

$$
\mathbf{U}(t)=\int_{-0.5}^{+0.5} \exp (i 2 \pi \omega t) \mathrm{d} \mathbf{Z}(\omega),
$$

where $d \mathbf{Z}(\omega)=\left[d Z_{X}(\omega), d Z_{Y}(\omega)\right]^{\prime}$ is a zero-mean bivariate random increment process that satisfies $\operatorname{cov}[d \mathbf{Z}(\omega)$, $d \mathbf{Z}(\lambda)]=$ $\delta(\omega-\lambda) \mathbf{f}(\omega) \mathrm{d} \omega \mathrm{d} \lambda$ and $\mathbf{f}(\omega)$ is the $2 \times 2$ spectral matrix whose diagonal elements, $f_{X X}(\omega)$ and $f_{Y Y}(\omega)$, are the auto-spectra and off-diagonal element $f_{X Y}(\omega)$ is the cross-spectrum. Cross-coherence between signals $X(t)$ and $Y(t)$ at a single frequency $\omega$, denoted by $\rho_{X Y}(\omega)$ can be expressed as

$$
\begin{aligned}
\rho_{X Y}(\omega) & =\left|\operatorname{cor}\left[d Z_{X}(\omega), d Z_{Y}(\omega)\right]\right|^{2} \\
& =\frac{\left|f_{X Y}(\omega)\right|^{2}}{\left[f_{X X}(\omega) f_{Y Y}(\omega)\right]} .
\end{aligned}
$$

Under the Cramér representation for stationary processes, the random coefficients $\mathrm{dZ}(\omega)$ for the different oscillatory components are uncorrelated. However, as a number of empirical results suggest, this is not reasonable for many brain signals. To extract more complex dependence structures in brain signals, one can use dual-frequency coherence which is developed under the class of harmonizable processes introduced by Loéve (1955). A process $\mathbf{U}(t)$ is harmonizable if it admits the representation

$$
\mathbf{U}(t)=\int_{-0.5}^{+0.5} \exp (i 2 \pi \omega t) \mathrm{d} \mathbf{Z}(\omega),
$$

where $\mathbf{d} \mathbf{Z}(\omega)$ is a zero-mean random vector increment process that satisfies

$$
\operatorname{cov}\left[\mathrm{d} \mathbf{Z}(\omega), \mathrm{d} \mathbf{Z}^{*}(\lambda)\right]=\mathbf{f}(\omega, \lambda) \mathrm{d} \omega \mathrm{d} \lambda,
$$

where $\mathbf{A}^{*}$ denotes the complex conjugate transpose of $\mathbf{A}$ and $\mathbf{f}(\omega, \lambda)$ is a complex-valued generalized spectral matrix or Loève spectral matrix. Under the representation in (4) we define dual-frequency auto-coherence and dual-frequency cross-coherence to be respectively,

$$
\begin{aligned}
& \rho_{X X}(\omega, \lambda)=\left|\operatorname{cor}\left[\mathrm{d} Z_{X}(\omega), \mathrm{d} Z_{X}(\lambda)\right]\right|^{2} \\
& \rho_{X Y}(\omega, \lambda)=\left|\operatorname{cor}\left[\mathrm{d} Z_{X}(\omega), \mathrm{d} Z_{Y}(\lambda)\right]\right|^{2} .
\end{aligned}
$$

Both $\rho_{X X}(\omega, \lambda)$ and $\rho_{X Y}(\omega, \lambda)$ fall on $[0,1]$. When $\rho_{X Y}(\omega, \lambda)$ is close to 1 , the real and imaginary parts of $\mathrm{d} Z_{X}(\omega)$ and $\mathrm{d} Z_{Y}(\lambda)$ are highly correlated. The procedure for estimating $\rho_{X X}(\omega, \lambda)$ and $\rho_{X Y}(\omega, \lambda)$ from several time series recorded from repeated trials is discussed in Section 4.

In our analysis, we noted that $\rho_{X X}(\omega, \lambda)$ and $\rho_{X Y}(\omega, \lambda)$ do not necessarily capture all the interesting features in the data. In particular, these two measures did not detect differences in the dependence structure between two conditions (one where the monkey correctly identified the correspondence and the other where the monkey made the mistake). Here, we will also develop other exploratory tools for probing dependence in the spectral domain. While dual-frequency coherence measures correlation between the complex-valued random coefficients, we shall also examine correlations between magnitudes of the random coefficients. The analogues of dual-frequency coherence measures in (6) are the dual-frequency auto-coherency and dual-frequency crosscoherency in amplitude which we define to be respectively,

$$
\begin{aligned}
& \zeta_{X X}(\omega, \lambda)=\operatorname{cor}\left[\left|\mathrm{d} Z_{X}(\omega)\right|,\left|\mathrm{d} Z_{X}(\lambda)\right|\right], \\
& \zeta_{X Y}(\omega, \lambda)=\operatorname{cor}\left[\left|\mathrm{d} Z_{X}(\omega)\right|,\left|\mathrm{d} Z_{Y}(\lambda)\right|\right] .
\end{aligned}
$$

Both $\zeta_{X X}(\omega, \lambda)$ and $\zeta_{X Y}(\omega, \lambda)$ fall on $[-1,1]$. When $\zeta_{X Y}(\omega, \lambda)$ is close to 1 or -1 , the magnitudes of $\mathrm{d} Z_{X}(\omega)$ and $\mathrm{d} Z_{Y}(\lambda)$ are highly correlated. 
In this section, both dual-frequency coherence and dual-frequency coherency in amplitude are contemporaneous measures of dependence between oscillatory waveforms. In the next section, we develop a model that addresses dependence between oscillatory waveforms at different time blocks.

For harmonizable processes, signals are represented as superpositions of sine and cosine waves at different frequencies $\omega$ with coefficients $\mathrm{d} Z(\omega)$ that may be correlated. Under this representation the signals are generally nonstationary. The class of harmonizable processes includes a large class of nonstationary processes by generalizing the covariance of the random coefficients $\mathrm{d} Z(\omega)$ to the Loève spectral matrix $\mathbf{f}(\omega, \lambda)$ from a diagonal matrix to one with potentially nonzero off-diagonal elements. The nonstationarity structure in the signals is evident when we consider the inverse Fourier transform of the Loève spectral matrix,

$$
\begin{aligned}
\Gamma(s, t) & =\mathbb{E}\left[\mathbf{U}(s) \mathbf{U}^{*}(t)\right] \\
& =\iint \exp \{2 \pi i(s \omega-t \lambda)\} \mathbf{f}(\omega, \lambda) \mathrm{d} \omega \mathrm{d} \lambda,
\end{aligned}
$$

where the temporal correlation function, $\Gamma$, describes directly the nonstationarity properties of the signals.

\section{PIECEWISE HARMONIZABLE PROCESSES}

We shall now develop the piecewise harmonizable process and extend the concepts of dual-frequency coherence and dual-frequency coherency in amplitude to describe frequency associations at different time blocks. To motivate this model, suppose that based on physiological information from an experiment, one can segment the time series into $B$ blocks which we denote $I_{1}, \ldots, I_{B}$ and define the block indicator function to be $\chi^{b}(t)$, which takes the value 1 when $t \in I_{b}$ and 0 otherwise. For the LFP data described in Section 5 , it was natural to segment the time series into $B=4$ time blocks where each block lasted for 512 milliseconds. During the first time block the monkey fixated at the centre of the screen; during the second block, one picture was presented and remained on the screen; during the third time block the picture was removed from the screen; and during the fourth time block four doorways were presented and the monkey needed to select one doorway that was matched to the picture presented on the second time block.

Based on the predefined block segmentation, the bivariate time series $\mathbf{U}(t)=[X(t), Y(t)]$ is piecewise harmonizable if it has the representation

$$
\mathbf{U}(t)=\sum_{b=1}^{B} \chi^{b}(t) \int_{-0.5}^{+0.5} \exp (i 2 \pi \omega t) \mathrm{d} \mathbf{Z}^{b}(\omega),
$$

where $d \mathbf{Z}^{b}(\omega)=\left[d Z_{X}^{b}(\omega), d Z_{Y}^{b}(\omega)\right]^{\prime}$ is a zero-mean random bivariate increment processes. An obvious special case of the piecewise harmonizable process, when $B=1$, is the harmonizable process already discussed in the previous section. Dependence measures of interest will include contemporaneous dual-frequency coherence and coherency in amplitude and their corresponding block-lagged dependence measures. In particular, the dual-frequency auto- and cross-coherence between frequencies $\omega$ at time block $b$ and $\lambda$ at time block $q$ are defined to be

$$
\begin{aligned}
& \rho_{X X}[(b, \omega) ;(q, \lambda)]=\left|\operatorname{cor}\left[\mathrm{d} Z_{X}^{b}(\omega), \mathrm{d} Z_{X}^{q}(\lambda)\right]\right|^{2}, \\
& \rho_{X Y}[(b, \omega) ;(q, \lambda)]=\left|\operatorname{cor}\left[\mathrm{d} Z_{X}^{b}(\omega), \mathrm{d} Z_{Y}^{q}(\lambda)\right]\right|^{2} .
\end{aligned}
$$

Similarly, these measures of dependence between lagged blocks can be extended to the auto- and cross-coherency in amplitudes which we define respectively, to be

$$
\begin{aligned}
& \zeta_{X X}[(b, \omega) ;(q, \lambda)]=\operatorname{cor}\left[\left|\mathrm{d} Z_{X}^{b}(\omega)\right|,\left|\mathrm{d} Z_{X}^{q}(\lambda)\right|\right] \\
& \zeta_{X Y}[(b, \omega) ;(q, \lambda)]=\operatorname{cor}\left[\left|\mathrm{d} Z_{Y}^{b}(\omega)\right|,\left|\mathrm{d} Z_{Y}^{q}(\lambda)\right|\right]
\end{aligned}
$$

The proposed lagged measures allow the investigator to study how changes in the oscillatory activity at frequency $\omega$ at a time block $b$ may be associated to an increase or a decrease in oscillatory activity at frequency $\gamma$ at the next time block $b+1$. Note that due to the temporal sequence in the data (i.e. activity at time block $b$ happens before $b+1$ ), one can make stronger claims of Granger-type causality. Thus, when $\zeta_{x y}[(b, \omega) ;(b+1, \lambda)]$ is close to 1 , then we say that an increase in magnitude of the $\omega$-waveforms in time-series $X$ at frequency $\omega$ at time block $b$ leads to an increase in the magnitude of the $\lambda$-waveforms in time-series $Y$ at the next time block $b+1$.

\section{ESTIMATION PROCEDURES AND INFERENCE}

We now present estimators for the coherence measures described before. We develop these estimators under the context of replicated time series. In our dataset which is typical in neuroscience experiments, we collect time series over hundreds of trials. Here, 
we shall assume that the spectral features are shared by signals recorded over all trials. For simplicity and to keep our focus on the new model and new dependence measures proposed, we shall also assume the trials to be independent realizations of the same underlying process.

Let $\mathbf{U}^{r}(t)=\left[X^{r}(t), Y^{r}(t)\right]$ be the zero mean time series recorded for the $r$ th trial $r=1, \ldots, R$. Our estimators are based on the Fourier coefficients which are the sample analogues of the random increment processes $\mathrm{d} Z_{X}(\omega), \mathrm{d} Z_{Y}(\omega)$,

$$
\mathrm{d}_{X}^{r}(\omega)=\sum_{t=0}^{T} X^{r}(t) \exp (-i 2 \pi \omega t) \quad \text { and } \quad \mathrm{d}_{Y}^{r}(\omega)=\sum_{t=0}^{T} Y^{r}(t) \exp (-i 2 \pi \omega t) .
$$

For the lagged linear measures estimators, we will use the block-dependent Fourier coefficients,

$$
\mathrm{d}_{X}^{r}(b, \omega)=\sum_{t=0}^{T} \chi^{b}(t) X^{r}(t) \exp (-i 2 \pi \omega t), \quad \mathrm{d}_{Y}^{r}(b, \omega)=\sum_{t=0}^{T} \chi^{b}(t) Y^{r}(t) \exp (-i 2 \pi \omega t) .
$$

Usually, the interest in interactions between frequencies of brain signals relies on frequency bands rather than singleton frequencies. In our analysis, we were primarily interested in the theta $(4-8 \mathrm{~Hz})$ and gamma $(30-50 \mathrm{~Hz})$ bands. Therefore, we summarize the Fourier coefficients at a particular frequency band, for instance $\vartheta$, by taking the average over all Fourier coefficients at frequencies that fall in the $\vartheta$-band, that is,

$$
d_{X}^{r}(\vartheta)=\operatorname{ave}\left\{d_{X}^{r}\left(\omega_{\ell}\right): \omega_{\ell} \in \vartheta \text {-band }\right\}, \quad d_{X}^{r}(b, \vartheta)=\operatorname{ave}\left\{d_{X}^{r}\left(b, \omega_{\ell}\right): \omega_{\ell} \in \vartheta \text {-band }\right\} .
$$

Similarly, for the estimators for the coherency in amplitude measures, we compute the average over the amplitudes:

$$
\left\|d_{X}^{r}(\vartheta)\right\|=\operatorname{ave}\left\{\left|d_{X}^{r}\left(\omega_{\ell}\right)\right|: \omega_{\ell} \in \vartheta \text {-band }\right\}, \quad\left\|d_{X}^{r}(b, \vartheta)\right\|=\operatorname{ave}\left\{\left|d_{X}^{r}\left(b, \omega_{\ell}\right)\right|: \omega_{\ell} \in \vartheta \text {-band }\right\} .
$$

Our estimators for the dual-frequency lagged auto-coherence and lagged cross-coherence are respectively,

$$
\begin{aligned}
& \hat{\rho}_{X X}[(b, \theta),(q, \gamma)]=\frac{\sum_{r=1}^{R} \mathrm{~d}_{X}^{r}(b, \theta) \mathrm{d}_{X}^{r *}(q, \gamma)}{\sum_{r=1}^{R}\left|\mathrm{~d}_{X}^{r}(b, \theta)\right| \sum_{r=1}^{R}\left|\mathrm{~d}_{X}^{r}(q, \gamma)\right|}, \\
& \hat{\rho}_{X Y}[(b, \theta),(q, \gamma)]=\frac{\sum_{r=1}^{R} \mathrm{~d}_{X}^{r}(b, \theta) \mathrm{d}_{Y}^{r *}(q, \gamma)}{\sum_{r=1}^{R}\left|\mathrm{~d}_{X}^{r}(b, \theta)\right| \sum_{r=1}^{R}\left|\mathrm{~d}_{Y}^{r}(q, \gamma)\right|} .
\end{aligned}
$$

For dual-frequency coherency in amplitude, the estimators are the sample correlations of the samples $\left\|d_{X}^{r}(b, \theta)\right\|$ and $\left\|d_{Y}^{r}(q, \gamma)\right\|$, $r=1, \ldots, R$. The estimators for the lagged dual-frequency auto-coherence in amplitude and cross-coherence are respectively,

$$
\begin{aligned}
& \hat{\zeta}_{X X}[(b, \theta) ;(q, \gamma)]=\frac{\sum_{r=1}^{R}\left(\left\|\mathrm{~d}_{X}^{r}(b, \theta)\right\|-\overline{\left\|\mathrm{d}_{X}^{r}(b, \theta)\right\|}\right)\left(\left\|\mathrm{d}_{X}^{r}(q, \gamma)\right\|-\overline{\left\|\mathrm{d}_{X}^{r}(q, \gamma)\right\|}\right)}{\sqrt{\sum_{r=1}^{R}\left(\left\|\mathrm{~d}_{X}^{r}(b, \theta)\right\|-\overline{\left\|\mathrm{d}_{X}^{r}(b, \theta)\right\|}\right)^{2} \sum_{r=1}^{R}\left(\left\|\mathrm{~d}_{X}^{r}(q, \gamma)\right\|-\overline{\left\|\mathrm{d}_{X}^{r}(q, \gamma)\right\|}\right)^{2}}}, \\
& \hat{\zeta}_{X Y}[(b, \theta) ;(q, \gamma)]=\frac{\sum_{r=1}^{R}\left(\left\|\mathrm{~d}_{X}^{r}(b, \theta)\right\|-\overline{\left\|\mathrm{d}_{X}^{r}(b, \theta)\right\|}\left(\left\|\mathrm{d}_{Y}^{r}(q, \gamma)\right\|-\overline{\left\|\mathrm{d}_{Y}^{r}(q, \gamma)\right\|}\right)\right.}{\sqrt{\sum_{r=1}^{R}\left(\left\|\mathrm{~d}_{X}^{r}(b, \theta)\right\|-\overline{\left\|\mathrm{d}_{X}^{r}(b, \theta)\right\|}\right)^{2} \sum_{r=1}^{R}\left(\left\|\mathrm{~d}_{Y}^{r}(q, \gamma)\right\|-\overline{\left\|\mathrm{d}_{Y}^{r}(q, \gamma)\right\|}\right)^{2}}} .
\end{aligned}
$$

To perform inference in the proposed measures of dependence, we are primarily interested in testing the null hypotheses

$$
H_{0}: \quad \rho_{X Y}[(b, \theta) ;(b-1, \gamma)]=0
$$

and

$$
H_{0}: \quad \rho_{X X}[(b, \theta) ;(b-1, \gamma)]=0 .
$$

The exact distribution for dual-frequency coherence estimators is inherited from the magnitude-squared coherence (MSC) function derived by Fisher (1928) and later further developed by Carter et al. (1973) and Brillinger (1981, 2001). However, under the null hypothesis the exact distribution is reduced to the following beta distribution:

$$
\operatorname{beta}(1, R-1): \quad p\left(\hat{\rho}^{2}\right)=(R-1)\left(1-|\hat{\rho}|^{2}\right)^{R-2} .
$$

We use this beta distribution to perform the inference of dual coherence measures in the data analysis section. Similarly, for the lagged coherence in amplitude measures, we test the hypotheses

$$
H_{0}: \quad \zeta_{X Y}[(b, \theta) ;(b-1, \gamma)]=0 \quad \text { and } \quad H_{0}: \quad \zeta_{X X}[(b, \theta) ;(b-1, \gamma)]=0 .
$$

In this case, we can use the distribution properties of the sample correlation which are based on the asymptotic normal distribution of Fisher's transformation (Ferguson, 1996):

$$
\sqrt{R}\left[\frac{1}{2} \log \frac{1+\hat{\zeta}}{1-\hat{\zeta}}-\frac{1}{2} \log \frac{1+\zeta}{1-\zeta}\right] \rightarrow \mathrm{N}(0,1)
$$




\section{ANALYSIS OF LFP DATA}

LFPs are obtained from electrodes located deep in the brain and sample the actual structures of interest with high spatial resolution. LFPs are similar to electroencephalograms (EEGs) as both signals measure brain electrophysiological activity. However, contrary to LFPs, scalp EEGs are recordings of a summation of electrical activity at low spatial resolution. Moreover, EEGs are attenuated by the skull, scalp and muscle artefacts. Finally, EEGs are quite poor at localizing activity deep and central in the brain such as that arising from medial structures as the Hc and NAc. Hence, our LFP data allow unique access to these deeply situated and critically important structures.

Our dataset consists of LFPs recorded while a macaque monkey was performing a learning association task. In the experiment, the monkey was presented either a novel or familiar picture from a set of four possible pictures each of which corresponded to a specific doorway. The monkey has to learn the correct correspondence over the course of the experiment. If the monkey chooses the correct door via joystick movement, a reward (juice or beverage) was given. Otherwise, no reward was given.

The LFP signals were recorded at the sampling rate of $1000 \mathrm{~Hz}$. Each trial consisted of $T=2048$ time points recorded over 2048 milliseconds. As previously discussed, each trial was segmented into $B=4$ time blocks each of which lasted for 512 milliseconds. The segmentation was defined by the experimental design. In the first time block, a cross-fixation was presented followed by the presentation of a picture in the second time block. In the third time block, the picture was removed leaving a time period of 512 milliseconds without visual stimulus during which the monkey was believed to be processing the visual information. In the last time block, four doors were presented. One of these doors was associated with the picture presented at the third time block and it was the monkey's task to identify the correct association. The image with the four doors was held on for 512 milliseconds to give the monkey a time to choose the door. The time course of each trial is graphically described in Figure 1.

We grouped the trials into the 'correct' and 'incorrect' responses. In the former, the monkey selected the correct correspondence (correct door). We considered a total of $R=215$ trials corresponding to a correct selection and $R=233$ corresponding to an incorrect selection. One of our main goals is to determine if the brain functional connectivity structure between the Hc and the NAc differs between the correct and incorrect responses. To achieve this, we performed an exploratory data analysis on the described LFPs to investigate spectral dependence structure between the Hc and NAc at theta and gamma frequency bands for each-time series trial set and compares the results for the correct and incorrect trials. The two components of the bivariate time series represent electrical activity at the $\mathrm{Hc}$ and NAc channels. As a preprocessing step, we applied a band-pass filter for each trial at (0.1, 50) $\mathrm{Hz}$ and then standardized each trial LFP to have global mean zero and unit variance.

For each condition, that is, correct and incorrect trials, we began by estimating the following dual-frequency coherence measures:

$$
\rho_{\mathrm{Hc}, \mathrm{Hc}}[(b, \theta) ;(q, \gamma)], \quad \rho_{\mathrm{NAc}, \mathrm{NAc}}[(b, \theta) ;(q, \gamma)], \quad \rho_{\mathrm{Hc}, \mathrm{NAc}}[(b, \theta) ;(q, \gamma)], \quad \rho_{\mathrm{Hc}, \mathrm{NAc}}[(b, \gamma) ;(q, \theta)],
$$

where $b, q$ are the indicators of time blocks, $b, q \in\{1,2,3,4\}$. In Figure 2, we displayed the dual-frequency coherence values that were statistically significantly different from zero. To account for multiple comparisons in this exploratory data analysis, we use the false discovery rate procedure of Benjamini and Hochberg (1995) at the 0.05 level. We observe that the dual-frequency coherence values are similar for correct and incorrect trials. In particular, the coherence values that exceed the threshold of 0.05 are common to the two conditions. These common associations are represented in Figure 3, where we noted that the gamma oscillation at the NAc region at adjacent blocks are highly related. Also, from these results we infer that increased oscillatory activity at gamma band in the NAc leads to increased oscillatory activity at theta band in NAc at the time period when the monkey had to make a selection of the door with the picture presented previously. This association is found in both correct and incorrect trials.

To explore spectral interactions further, we computed estimates for the amplitude coherency quantities:

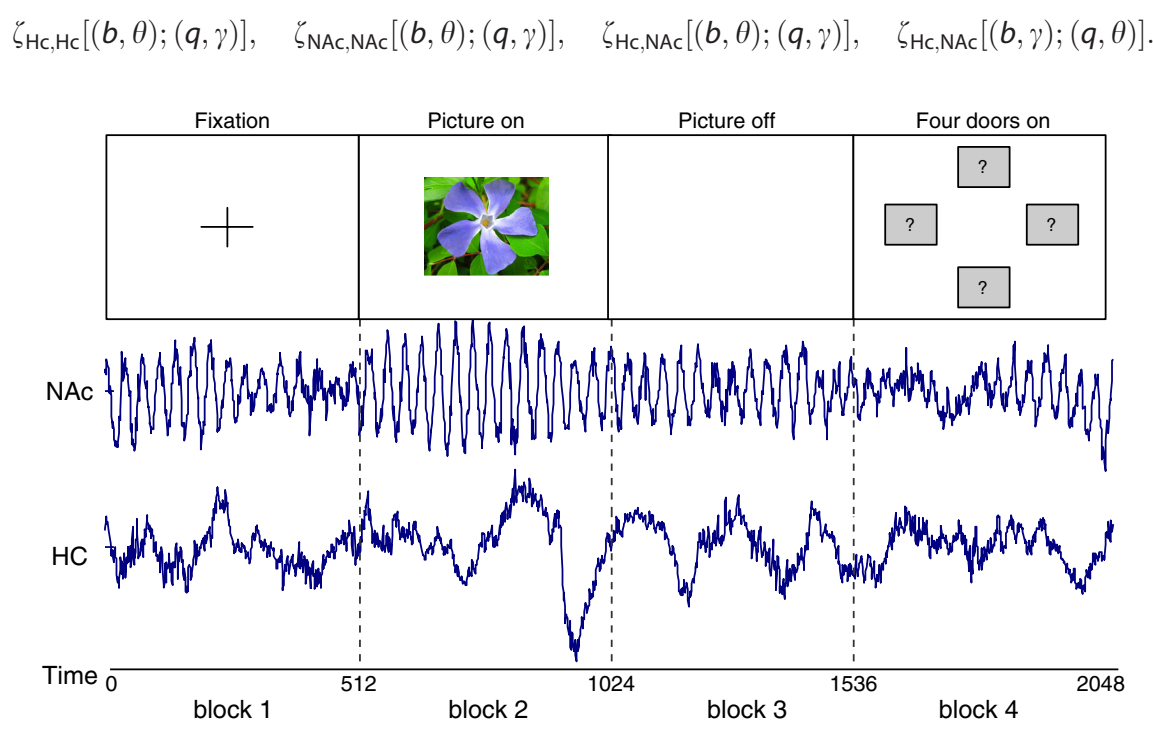

Figure 1. Time course of trials. Each block length consists on 512 time points 

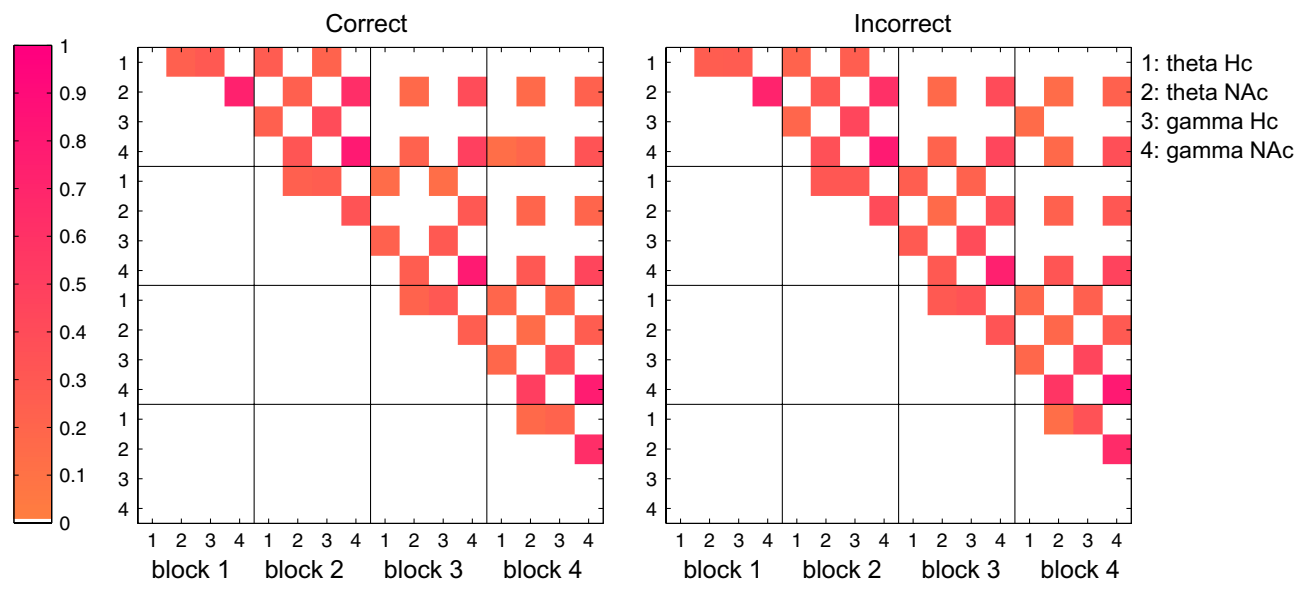

Figure 2. In colour scale, we show the squared root of the dual-frequency coherence values significantly different from zero (false discovery rate correction at 0.05 level). By symmetry, we focused only on the upper diagonal matrix. The lines in the matrix define the time blocks and the code for the matrix elements is described at the right

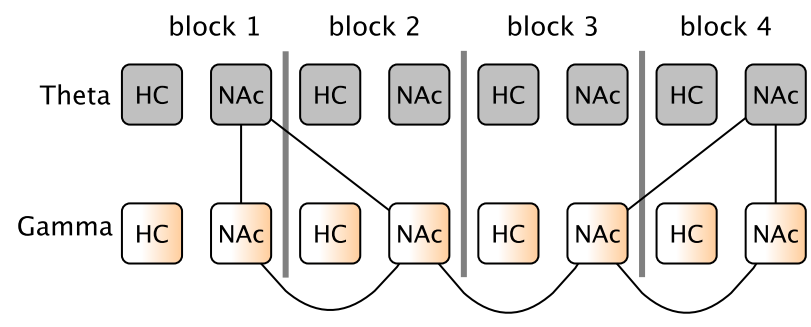

Figure 3. Coherence links that were significantly different from zero and greater than the threshold of 0.5 . These correlations were common in the correct and incorrect trials
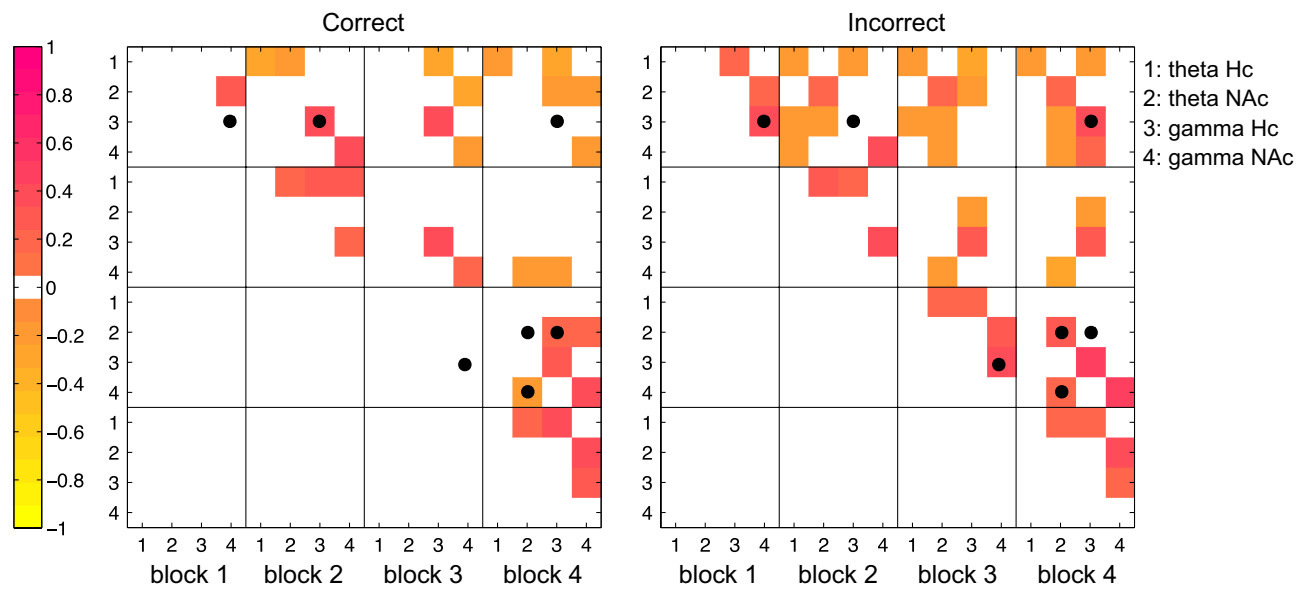

Figure 4. Significant values of the coherency in amplitude measures. In colour scale are showed the frequency coherencies in amplitude values significantly different from zero (to correct for multiple comparison we used false discovery rate correction at 0.05 level). By symmetry, we focus only on the upper diagonal matrix. The lines in the matrix define the time blocks and the code for the matrix elements is described at the right. Black dots indicate that there is a significant difference between the correct and incorrect trials (false discovery rate correction at 0.05 level)

Coherencies in amplitude values that are significantly different from zero are displayed in colour scale in Figure 4. To account for multiple comparisons, we use the false discovery rate procedure at the 0.05 level. We determined statistically significant differences in cross-oscillatory coherency in magnitude for the correct and incorrect trials; these are indicated with a black dot in Figure 4. The numerical values of coherencies in amplitude, where a significant difference was found between correct and incorrect trials, are displayed in Table 1. A graphical representation of the links at which these significant differences were present is displayed in Figure 5. 
Table 1. Coherency in amplitude values where a significant difference was found between correct and incorrect trials

\begin{tabular}{|c|c|c|}
\hline & Correct & Incorrect \\
\hline$\hat{\zeta}_{\mathrm{HC} . \mathrm{NAC}}([1, \gamma] ;[1, \gamma])$ & 0.023 & 0.357 \\
\hline $\mathrm{\zeta}_{\mathrm{HC} . \mathrm{HC}}([1, \gamma] ;[2, \gamma])$ & 0.408 & 0.147 \\
\hline$\zeta_{\mathrm{HCHC}}([1, \gamma] ;[4, \gamma])$ & 0.054 & 0.380 \\
\hline${ }_{N}^{N A C, N A c}([3, \theta] ;[4, \theta])$ & 0.017 & 0.296 \\
\hline $\mathrm{NAc}_{\mathrm{NC}}([3, \theta] ;[4, \gamma])$ & 0.219 & -0.070 \\
\hline${ }^{C} \mathrm{Hc}, \mathrm{NAc}([3, \gamma] ;[3, \gamma])$ & 0.081 & 0.351 \\
\hline$\hat{\zeta}_{\text {NAc,NAc }}([3, \gamma] ;[4, \theta])$ & -0.185 & 0.186 \\
\hline
\end{tabular}

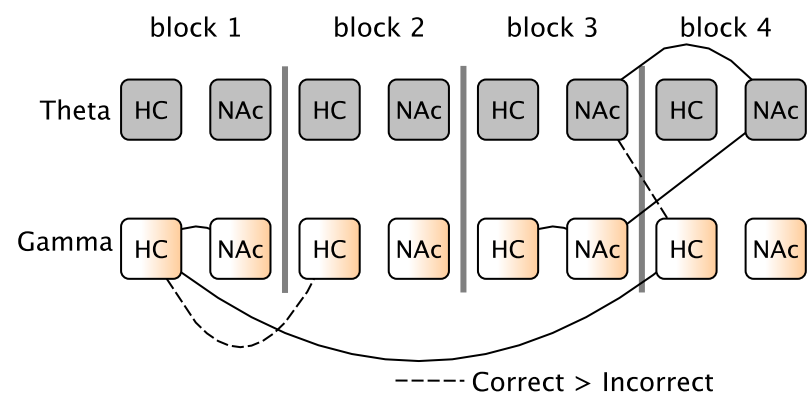

Figure 5. Coherency in amplitude links at which there is a significant difference between the correct and incorrect trials

It is interesting that the cross-dependence between the amplitudes of theta activity in NAc during time block $b=3$ (picture off) and the amplitudes of gamma activity in Hc during time block $b=4$ (doorways presented) for the correct trials are greater than that for the incorrect trials. This result suggests that $\zeta_{\mathrm{NAc}, \mathrm{Hc}}[(3, \theta) ;(4, \gamma)]$ could be an electrophysiological discriminator for the outcome of a trial (correct vs. incorrect). Another interesting result is that for correct trials, the amplitudes of theta activity in NAc during the time block $b=4$ (when the doorways were presented and the monkey was preparing for a response) are highly correlated with both the theta and gamma activity amplitudes in the NAc from the picture off time block.

\section{CONCLUSION}

We presented exploratory tools for determining whether increased oscillatory activity at some frequency band leads increased or decreased oscillatory activity at another frequency band. Our contributions are formal measures of dependence under the class of piecewise harmonizable processes. We developed these tools for replicated time series where each trial can be segmented, in a physiologically meaningful way, into disjoint time blocks. We presented an application to analyse theta and gamma frequency band interactions, in LFP signals recorded at the Hc and NAc and found some interesting results that show differences in brain functional connectivity between the correct and incorrect responses.

\section{Acknowledgements}

This research was supported in part by CONACyT Mexico (Gorrostieta), NSF DMS (Ombao), NSF SSE (Ombao and Prado) and the Conte Center Grant (Eskandar and Ombao).

\section{REFERENCES}

Benjamini, Y. and Hochberg, Y. (1995) Controlling the false discovery rate: a practical and powerful approach to multiple testing. Journal of the Royal Statistical Society, Series B (Methodological) $\mathbf{5 7}, \mathbf{2 8 9 - 3 0 0 .}$

Bramon, E., Rabe-Hesketh, S., Sham, P., Murray, R. M. and Frangou, S. (2004) Meta-analysis of the P300 and P50 waveforms in schizophrenia. Schizophrephia Research 70, 315-29.

Brillinger, D. (1981, 2001) Time Series: Data Analysis and Theory, 2nd edn. San Francisco: Holden-Day. Republished in 2001 by the Society for Industrial and Applied Mathematics, Philadelphia, PA.

Carter, G. C., Knapp, C. H. and Nuttall, A. H. (1973) Estimation of the magnitude-squared coherence function Via overlapped fast Fourier transform processing. IEEE Transactions on Audio and Electroacoustics 21, 337-44.

Ferguson, T. S. (1996) A Course in Large Sample Theory. Texts in Statistical Science. Boca Raton, Florida: Chapman \& Hall/CRC.

Fiecas, M. and Ombao, H. (2011) The Generalized shrinkage estimator for the analysis of functional connectivity of brain signals. Annals of Applied Statistics 5, 1102-25.

Fiecas, M., Ombao, H., Linkletter, C., Thompson, W. and Sanes, J. N. (2010) Functional connectivity: shrinkage estimation and randomization test. Neurolmage 40, 3005-14. 
Fisher, R. A. (1928) The general sampling distribution of the multiple correlation coefficient. Proceedings of the Royal Society of London 21, 654-73. Händel, B. and Haarmeier, T. (2009) Cross-frequency coupling of brain oscillations indicates the success in visual motion discrimination. Neurolmage 45, 1040-46.

Loéve, M. (1955) Probability Theory. The University Series in Higher Mathematics. Canada: D. Van Nostrand Company.

Nyhus, E. and Curran, T. (2010) Functional role of gamma and theta oscillations in episodic memory. Neuroscience and Biobehavioral Reviews 34, 1023-35.

Ombao, H. and Van Bellegem, S. (2008) Coherence analysis: a linear filtering point of view. IEEE Transactions on Signal Processing 56, 2259-66.

Shumway, R. and Stoffer, D. (2011) Time Series Analysis and its Applications, 3rd edn. New York: Springer.

Sun, F., Miller, L. and Desposito, M. (2004) Measuring interregional functional connectivity using coherence and partial coherence analyses of fMRI data. Neurolmage, 21, 647-58.

Trejo, L. J., Knuth, K., Prado, R., Rosipal, R., Kubitz, K., Kochavi, R., Matthews, B. and Zhang, Y. (2007) EEG-based estimation of mental fatigue: convergent evidence for a three-state model. In Augmented Cognition, HCII LNAI (eds D. D. Schmorrow and L. M. Reeves). New York: Springer, pp. $201-11$.

Varela, F., Lachaux, J. P., Rodriguez, E. and Martinerie, J. (2001) The brain web: phase synchronization and large-scale integration. Nature Reviews 2 , 229-39. 